\title{
Excellent Luminous Efficacy and Color Homogeneity of White Light-Emitting Diodes with $\mathrm{YPO}_{4}: \mathrm{Ce}^{3+}: \mathrm{Tb}^{3+}$ Phosphor
}

\author{
Vo Phu Thoai, Nguyen Doan Quoc Anh \\ Faculty of Electrical and Electronics Engineering, Ton Duc Thang University, Ho Chi Minh City, Vietnam
}

\begin{abstract}
In this paper, we focus on researching the method, which the color homogeneity and the lumen output of multi-chip white LED lamps (MCW-LEDs) need to support for increasing the efficiency. The successful results can be achieved by mixing the green $\mathrm{YPO}_{4}: \mathrm{Ce}^{3+}: \mathrm{Tb}^{3+}$ phosphor with their phosphor compounding. Through experiment results, we assert that the MCW-LEDs can achieve the significant consequence in performance by following that method and it is also again confirmed that when the concentration of $\mathrm{YPO}_{4}: \mathrm{Ce}^{3+}: \mathrm{Tb}^{3+}$ has tendency to increase, which impulse the development of the color uniformity and the luminous efficacy of MCW-LEDs with average correlated color temperatures (CCT) of $8500 \mathrm{~K}$, while the color quality scale shows signs of gradual decline. It is not difficult to gain incredible presentation of MCW-LEDs if we are clever in choosing the suitable concentration and size of $\mathrm{YPO}_{4}: \mathrm{Ce}^{3+}: \mathrm{Tb}^{3+}$.
\end{abstract}

\section{Introduction}

In recent years, the study which scientists concerned the most is that howto enhance the light performance of white LED packages effectively and uninterruptedly. In which, the matter which concerned as the important one related to white LED lamps is lumen output and color [1-10]. After many efforts in experiments and researches, Anh with his colleague have finally recognized that when $\mathrm{SiO}_{2}$ particles is poured into YAG:Ce ${ }^{3+}$ phosphor compounding, it will give us an uncontemplated result [11]. The quality of multi-chip white LED lamps could significantly promote as possible through that process. In order to create a compounding which is able to absorb the blue light of the blue chips and emits the yellow light, the yellow YAG:Ce ${ }^{3+}$ phosphor material has to rely on the silicone glue to mix together for the creation of compounding. The white light is a part of component obtained from the process of mixture of the colors. As a result, the emitted nomal light tends to be more bluish when it comes to the surface of LED, while its position is in nearly parallel to the LED surface it will bring the tendency of more yellowish. Hence, if light intensity distributions is different, it can create a ring which is more likely yellow [12-20].

By using the method of optimization for phosphors or optical structures of white LEDs, it will help the white light quality and lumen output have a positive aspect. In addition, $\mathrm{YPO}_{4}: \mathrm{Ce}^{3+}: \mathrm{Tb}^{3+}$ particles is known as efficient green component of trichromatic lamp phosphor, which includes so many special features like high quantum efficiency, stability at high temperature and because of these features, it becomes more and more popular [21].
Moreover, $\mathrm{YPO}_{4}: \mathrm{Ce}^{3+}: \mathrm{Tb}^{3+}$ is applied particularly for very high-loading and long life-time fluorescent lamps. Therefore, it is one of the most popular commercialized oxide phosphors. In this paper, we definitely suggest and employ the green $\mathrm{YPO}_{4}: \mathrm{Ce}^{3+}: \mathrm{Tb}^{3+}$ phosphor in the phosphor compounding of MCW-LEDs to help color uniformity and lumen output attain the best result. The conducted research is a 3-stage process: (1) Constructing model of MCW-LEDs; (2) Putting $\mathrm{YPO}_{4}: \mathrm{Ce}^{3+}: \mathrm{Tb}^{3+}$ into the phosphor compounding of the simulated MCW-LEDs (3) Analyzing the effects of $\mathrm{YPO}_{4}: \mathrm{Ce}^{3+}: \mathrm{Tb}^{3+}$ concentration on the lighting performance, including the emission spectra and the scattering. The investigated results give us a conclusion that if green $\mathrm{YPO}_{4}: \mathrm{Ce}^{3+}: \mathrm{Tb}^{3+}$ and yellow YAG:Ce ${ }^{3+}$ phosphors are mixed together to be a perfect compounding, color uniformity and lumen output can be attained the best quality.

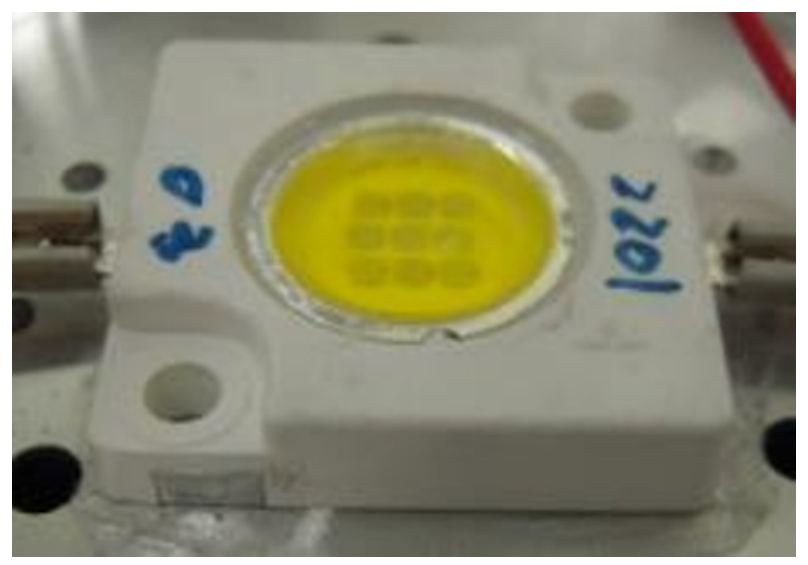

(a) 


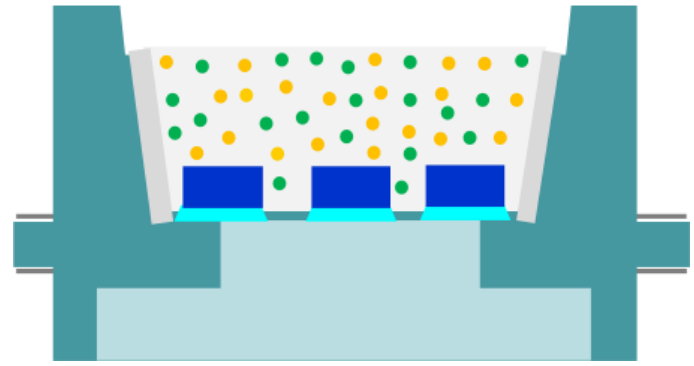

(b)

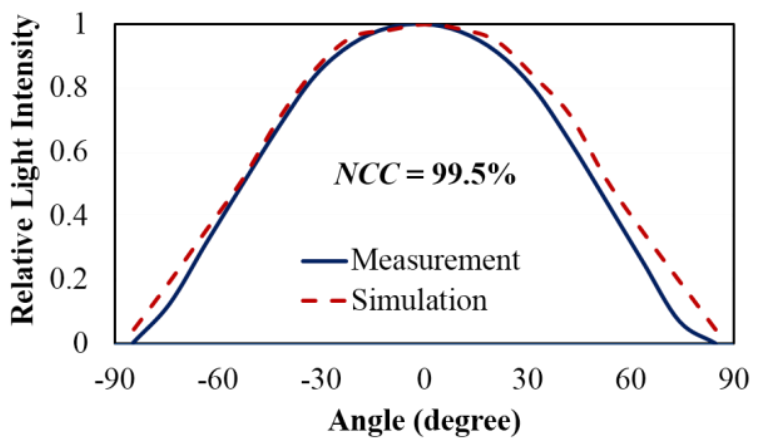

(c)

Fig. 1. (a) Photograph of an employed MCW-LED, (b) Incup phosphor geometry, (b) The calculation of normalized cross correlation $(N C C)$.

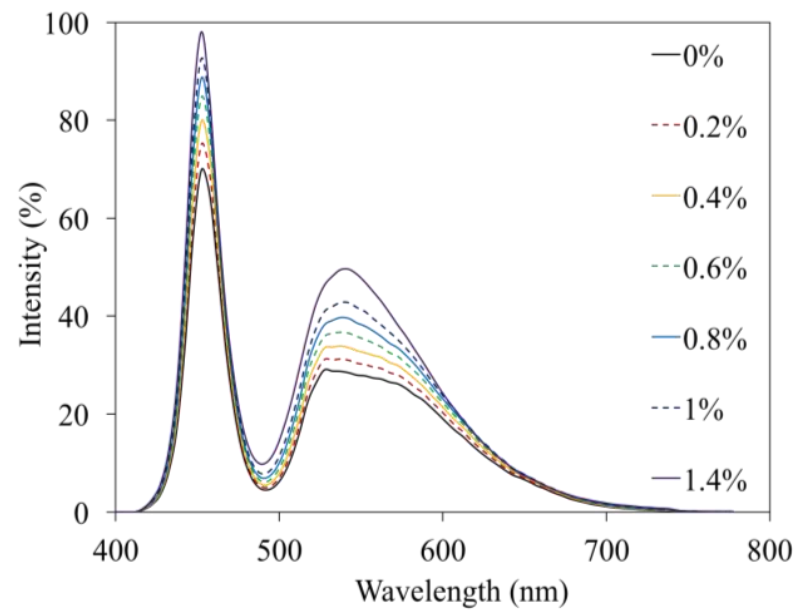

Fig. 2. Emission spectra of multiple phosphors.

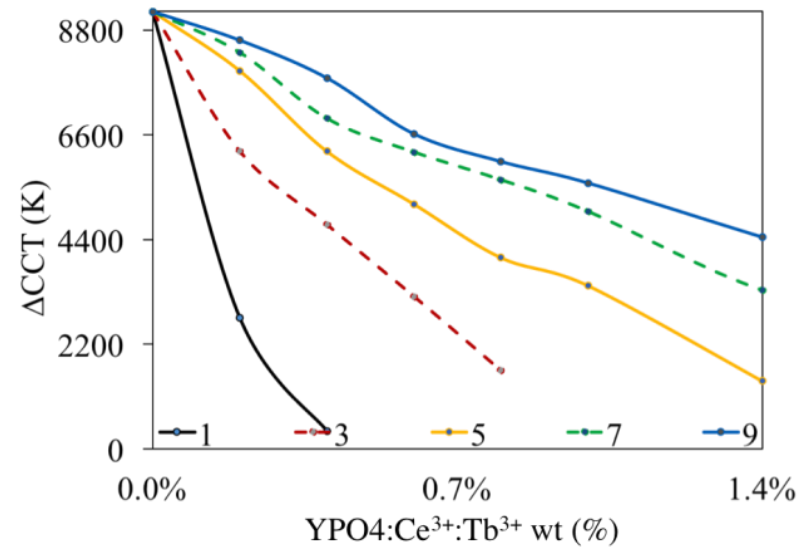

Fig. 3. The CCT peak-valley deviation as a function of the concentration and size of $\mathrm{YPO}_{4}: \mathrm{Ce}^{3+}: \mathrm{Tb}^{3+}$.

\section{Simulation and discussion}

According to LightTools 8.1.0 program and Monte Carlo method, the phosphor layer of real MCW-LEDs is carried out simulation with flat silicone layer. We carry out the modeling process through 2 main periods: (1) the mechanical structures and optical properties of MCWLED lamps must to be set and built. (2) Next, after completing the first step we continue monitoring the optical influences of phosphor compounding through the diversity of $\mathrm{YPO}_{4}: \mathrm{Ce}^{3+}: \mathrm{Tb}^{3+}$ concentration. The results of the NCC related experiments are shown in Fig. 1(c). The NCC values is higher than $99.5 \%$ for both CPG and IPG, so the WLED models are appropriate to be used in the computational simulation.

In order to understand the influence of $\mathrm{YAG}: \mathrm{Ce}^{3+}$ and $\mathrm{YPO}_{4}: \mathrm{Ce}^{3+}: \mathrm{Tb}^{3+}$ phosphor compounding on the performance of MCW-LED lamps, we must have been carried out some experiments. In there, in-cup phosphor compounding is considered to clarify, which has average CCTs at $8500 \mathrm{~K}$. In figure 1(top), there is a clearly description about MCW-LED lamps with conformal phosphor compounding having average CCTs of $8500 \mathrm{~K}$. It also indicates the simulation of MCW-LEDs, which the components do not contain $\mathrm{YPO}_{4}: \mathrm{Ce}^{3+}: \mathrm{Tb}^{3+}$. The reflector has a bottom length of $8 \mathrm{~mm}$, a height of 2.07 $\mathrm{mm}$ and a length of $9.85 \mathrm{~mm}$ at its top surface. The conformal phosphor compounding, whose thickness is fixed at $0.08 \mathrm{~mm}$, covers nine chips. Each LED chip with the square base area of $1.14 \mathrm{~mm}^{2}$ and the height of 0.15 $\mathrm{mm}$ is attached to the cavity of the reflector. The radiant flux of each blue chip is $1.16 \mathrm{~W}$ and $453 \mathrm{~nm}$ is the peak wavelength.

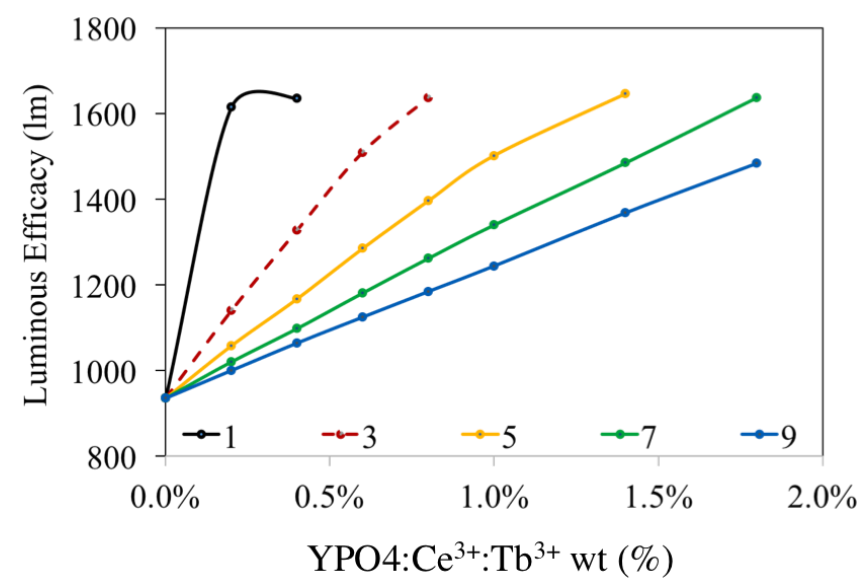

Fig. 4. Luminous efficacy as a function of the concentration and size of $\mathrm{YPO}_{4}: \mathrm{Ce}^{3+}: \mathrm{Tb}^{3+}$.

Figure 2 detailed displays the emission spectra of incup phosphor compounding with different ranges of $\mathrm{YPO}_{4}: \mathrm{Ce}^{3+}: \mathrm{Tb}^{3+}$ concentration and it is sometimes even up to $1.4 \%$. Through this chart, we can know clearer that the lumen output of MCW-LEDs may be enhanced after YPQ: $\mathrm{Ce}^{3+}: \mathrm{Tb}^{3+}$ particles are poured together into phosphor compound.

In this research, it is really important and necessary for meeting the demand of the LED product specification, so MCW-LED needs requesting to work in the scope of 
average CCTs of $8500 \mathrm{~K}$. In addition, when the concentration of the green YPQ: $\mathrm{Ce}^{3+}: \mathrm{Tb}^{3+}$ phosphor grows, that of yellow YAG: $\mathrm{Ce}^{3+}$ phosphor must be obviously reduced because of the maintaining the average CCTs of $8500 \mathrm{~K}$. The weight percentage of the LED phosphor layer can be expressed as:

$$
W_{p l}=W_{\text {yellow phosphor }}+W_{\text {silicone }}+W_{\text {green phosphor }}=100 \%
$$

In this formula, $W_{\text {silicone, }} W_{\text {yellowphosphor }}$ and $W_{\text {greenphosphor }}$ are the weight percentage of the silicone glue, the yellow YAG: $\mathrm{Ce}^{3+}$ phosphor and the green YPQ: $\mathrm{Ce}^{3+}: \mathrm{Tb}^{3+}$ phosphor in turn.

The simulation receives the results, which the angular color deviation of MCW-LED is in the appearance and absence of YPQ: $\mathrm{Ce}^{3+}: \mathrm{Tb}^{3+}$ are shown in Figure 3. It can be observed that the CCT peak-valley deviation reduces significantly with the involving of YPQ: $\mathrm{Ce}^{3+}: \mathrm{Tb}^{3+}$. It means that the spatial color distribution of MCW-LEDs with YPQ: $\mathrm{Ce}^{3+}: \mathrm{Tb}^{3+}$ is much flatter than that in lacking of YPQ: $\mathrm{Ce}^{3+}: \mathrm{Tb}^{3+}$.

If we just optimize an element, the optical system can weakly achieve in other aspects. In particular, we cannot achieve the best COS values and the efficiency of the white LED package at the same time, there is only aspect achieving results as desired. The answer is that if we need to collect a good CRI, it has to have a broad source spectrum and efficiency is maximized at monochromatic radiation with a wavelength of $555 \mathrm{~nm}$. In this study, CQS, luminous flux and CCT P-V deviation values are three competing properties. About the simulation results in Figure 4 and Figure 5, it can be found that the lumen output grows with the concentration of YPQ: $\mathrm{Ce}^{3+}: \mathrm{Tb}^{3+}$. Besides, the results also show that the more YPQ: $\mathrm{Ce}^{3+}: \mathrm{Tb}^{3+}$ concentration involved, the higher luminous flux we get, but the CQS tends to be less. Moreover, the concentration of YPQ: $\mathrm{Ce}^{3+}: \mathrm{Tb}^{3+}$ develop in a downward trend but it is insignificant, MCW-LED packages could be accomplished with better correlated color temperature uniformity and higher luminous flux.

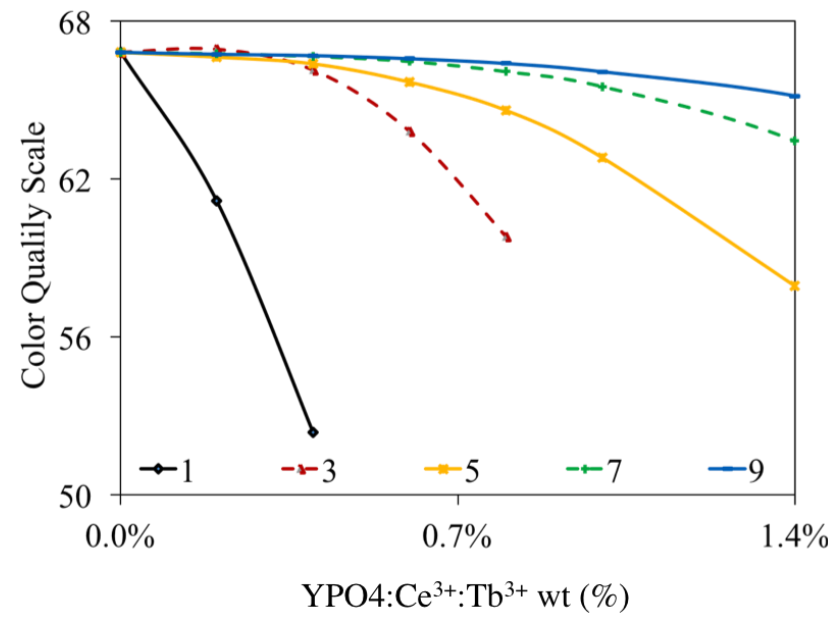

$\mathbf{F} \square$. 5. Color quality scale as a function of the concentration and size of YPQ: $\mathrm{Ce}^{3+}: \mathrm{Tb}^{3+}$.

\section{Conclusion}

In this study, the main purpose which all the paper we focus on study is the effect of the green YPQ: $\mathrm{Ce}^{3+}: \mathrm{Tb}^{3+}$ phosphor and its ability in improving the quality of both the color uniformity and the lumen output of white LED packages. Firstly, the color uniformity can be significantly enriched regardless of the average CCT value and the phosphor geometry if it obeys the Miescattering theory. This can be achieved due to the compensation of light-scattering to white LED packages. Next by applying Monte Carlo simulation, we demonstrated that the change of lumen output depends on the YPQ: $\mathrm{Ce}^{3+}: \mathrm{Tb}^{3+}$ concentration. Indeed, the lumen output grows with the YPQ: $\mathrm{Ce}^{3+}: \mathrm{Tb}^{3+}$ concentration. Finally, the paper proves the implication that YPQ: $\mathrm{Ce}^{3+}: \mathrm{Tb}^{3+}$ phosphor application is a promising solution for developing the pc-WLEDs, and for all the manufacture of white LED packages.

This research is funded by Department of Science and Technology of Binh Thuan province under grant number DA 02- 01- 2018.

\section{References}

1. C. Shen, J. Chu, F. Qian, X. Zou, C. Zhong, K. Li and S. Jin, J. Mod. Opt. 59, 1199 (2012).

2. S. Chawla, T. Roy, K. Majumder and A. Yadav, J. Exp. Nanosci. 9776 (2014).

3. M. S. Jang, Y. H. Choi, S. Wu, T. G. Lim and J. S. Yoo, J. Inf. Display 17, 117 (2016).

4. Y. H. Won, H. S. Jang, K. W. Cho, Y. S. Song, D. Y. Jeon and H. K. Kwon, Opt. Lett. 34, 1 (2009).

5. Z. M. Chen, S. X. Jiang, R. H. Guo, B. J. Xin and D. G. Miao, Materials Technology: Advanced Performance Materials 29, 198 (2014).

6. Z. M. Chen, S. X. Jiang, R. H. Guo, B. J. Xin and D. G. Miao, Materials Technology: Advanced Performance Materials, vol. 31 (1), pp. 7-12, 2016.

7. M. Zhang, X. -H. Li, Z. -X. Wang, Q. -Y. Hu, H. -J. Guo, Trans. Nonferrous Met. Soc. China 20115 (2010).

8. Z.-Y Liu, S. Liu, K. Wang, and X.-B Luo, Appl. Opt. 49, 247 (2010).

9. K.-C. Lee, S.-M. Kim and J.-H. Moon, In Proceedings of Tenth International Conference on Solid State Lighting, San Diego, USA, 18 August 2010: Vol 7784. Washington: SPIE, 2010.

10. J. -P. Kim, M. -S. Jang, W. -H. Kim, J. -Y. Joo, J. -H. Cho, D. -W. Kim, S. -B. Song, Opt. Mater. 34, 1614 (2012).

11. N. D. Q. Anh, M. F. Lai, H. Y. Ma, H. Y. Lee, “ J. Chin. Inst. Eng., vol. 38, 297 (2015).

12. L. Yang, Z.-C. Lv, Y. Jiaojiao and S. Liu, Appl. Opt. 52, 5539, 2013.

13. S. Wang, X. Chen, M. Chen, H. Zheng, H. Yang, and S. Liu, Appl. Opt. 53, 8492 (2014).

14. H. - Y. Lin, K. - J. Chen, S. -W. Wang, C. -C. Lin, K. -Y. Wang, J. -R. Li, P. -T. Lee, M. -H. Shih, X. 
Li, H. -M. Chen, and H.-C. Kuo, Opt. Exp. 23, 27 (2014).

15. S. Yu, Z. Li, G. Liang, Y. Tang, B. Yu, and K. Chen. Photonics Res. 4, 140 (2016).

16. F. W. Mont, J. K. Kim, M. F. Schubert, H. Luo, E. F. Schubert, and R. W. Siegel, J. Appl. Phys., 103, 083120 (2007).

17. H. -C. Chen, K. -J. Chen, C. -C. Lin, C. -H. Wang, H. -V. Han, H. -H. Tsai, H. -T. Kuo, S. -H. Chien, M. - H. Shih and H. -C. Kuo, Nanotechnology 23, 1 (2012).
18. M. F. Lai, N. D. Q. Anh, H. Y. Ma, H. Y. Lee, J. Chin. Inst. Eng. 39, 1 (2016).

19. N. D. Q. Anh, H. Y. Lee, T. T. Phuong, N. H. K. Nhan, T. H. Q. Minh and T. H. Ly, J. Chin. Inst. Eng, 40, 1 (2017).

20. N. D. Q. Anh and H. Y. Lee, J. Chin. Inst. Eng 39, 871 (2016).

21. Yen, W. M., and M. J. Weber. Inorganic Phosphors: Compositions, Preparation and Optical Properties. CRC Press, LLC, 2000 N.W. Corporate Blvd., Boca Raton, Florida 33431, 2004. 